\title{
Linx
}

Revue des linguistes de l'université Paris X Nanterre

38 | 1998

L'acquisition de la temporalité en situation bilingue

\section{Développement de la temporalité en turc chez des enfants bilingues (turc-français) en France}

The development of temporality in Turkish by Turkish-French bilingual children in France

Mehmet-Ali Akinci

\section{CpenEdition}

\section{Journals}

Édition électronique

URL : http://journals.openedition.org/linx/849

DOI : $10.4000 /$ linx.849

ISSN : 2118-9692

Éditeur

Presses universitaires de Paris Nanterre

Édition imprimée

Date de publication : 1 décembre 1998

Pagination : 19-34

ISSN : 0246-8743

Référence électronique

Mehmet-Ali Akinci, « Développement de la temporalité en turc chez des enfants bilingues (turc-

français) en France », Linx [En ligne], 38 | 1998, mis en ligne le 26 juin 2012, consulté le 02 mai 2019.

URL : http://journals.openedition.org/linx/849; DOI : 10.4000/linx.849

Ce document a été généré automatiquement le 2 mai 2019.

Département de Sciences du langage, Université Paris Ouest 


\section{Développement de la temporalité en turc chez des enfants bilingues (turc- français) en France}

The development of temporality in Turkish by Turkish-French bilingual children in France

\section{Mehmet-Ali Akinci}

1 Dans tout récit la tâche des narrateurs est d'établir une trame générale et de la suivre à travers leur narration afin de réaliser un ensemble cohérent. Dans un texte multipropositionnel, les événements doivent être organisés de manière hiérarchique les uns par rapport aux autres. Et pour cela, les narrateurs, qui disposent d'un ensemble de moyens linguistiques variés, sont libres dans leur choix. Ces moyens apparaissent chez les enfants au long du processus de développement, et il est d'autant plus intéressant d'étudier cette évolution lorsqu'il s'agit d'enfants bilingues utilisant deux systèmes temporels différents.

2 L'étude présentée dans cet article vise quelques aspects du développement de la temporalité en turc chez des enfants bilingues turc-français âgés de 5 à 10 ans issus de l'immigration turque à Grenoble, immigration essentiellement d'origine rurale venue en France au milieu des années 70. Pour la collecte de nos données, nous avons eu recours au livre sans textes composé d'images "Frog where are you?" de M. Mayer, (1969). Les enregistrements de cette histoire ont été effectués auprès de 93 enfants, en turc et en français. Les données ont été transcrites, analysées, puis comparées aux résultats d'AksuKoç (1994) qui travaille auprès de monolingues turcs de couche sociale élevée d'Istanbul, 
ainsi qu'à ceux d'Aarssen (1996) qui, lui, travaille auprès d'enfants bilingues turcs issus de l'immigration aux Pays-Bas.

3 Les questions auxquelles nous avons essayé d'apporter des réponses sont les suivantes:

- Comment font les enfants bilingues issus de l'immigration (milieu social défavorisé) pour organiser leur narration? Quels sont les temps dominants utilisés comme temps du récit? Quelles explications peut-on donner aux alternances temporelles?

- Quelle image du développement du turc chez ces enfants cela donne-t-il, et quelles hypothèses peut-on émettre quant à ce profil de développement? Quels peuvent en être les facteurs?

4 Afin de répondre à ces questions, nous présentons brièvement dans une première partie la langue turque et son système verbal, ensuite la méthodologie que nous avons adoptée pour mener cette étude. Dans une troisième partie, nous verrons les temps dominants relevés chez nos sujets. Le dernier point traitera des alternances temporelles. Nous conclurons notre étude en essayant d'établir des liens entre les résultats obtenus et des facteurs explicatifs.

\section{Quelques mots sur le turc}

$5 \quad$ Le turc ${ }^{1}$ appartient au sous-groupe altaïque du groupe de langues ouralo-altaïques.

6 Du point de vue morphologique, le turc est une langue agglutinante : tout fonctionne suivant un système de suffixation. Ainsi, chaque élément garde son identité sémantique et phonologique, ainsi que sa position dans le mot. Du point de vue syntaxique, l'ordre des constituants est S.O.V. Il s'agit d'un ordre canonique qui n'est pas rigide, puisque l'ordre des mots dans les phrases simples et les propositions principales connaît un haut degré de variation pragmatique, notamment pour signaler des points de vue contrastifs, la topicalisation, et l'arrière-plan (Erguvanli, 1984).

7 Du point de vue phonétique, le turc connait la règle de l'harmonie vocalique (assimilation de toute voyelle par la voyelle de la syllabe précédente) et l'assimilation de voisement ou consonantique (les consonnes sonores ne peuvent être suivies de consonnes sourdes, et vice versa).

8 La fonction du nom dans la phrase est marquée par des suffixes de cas qui permettent d'exprimer l'état du nom et ses déplacements dans l'espace et le temps. Au nombre de cinq, ceux-ci se divisent en deux groupes : les cas grammaticaux (l'accusatif et le génitif) et les cas spatiaux (le directif, le locatif, l'ablatif).

Le système verbal : sur le plan morphologique, le turc exprime les catégories du temps, de l'aspect et de la modalité, d'une part, en adjoignant le suffixe adéquat à la racine verbale qui est toujours invariable et d'autre part, par la sémantique des verbes et des adverbes. La règle fondamentale pour la formation des verbes est donc l'adjonction d'une série d'affixes à la racine tout en tenant compte des règles de l'harmonie vocalique et de l'assimilation consonantique. La forme verbale conjuguée peut ainsi être plus ou moins longue, comme le montre le schéma qui suit :

racine verbale + négation + suffixe de voix + suffixe aspecto-temporel + suffixe de personne

10 dans lequel des suffixes intermédiaires indiquent la valence, la voix (le causatif, le réciproque, le réflexif et le passif), la négation, la modalité (la possibilité, le déontique, l'hypothétique et l'optatif) ainsi que la force illocutoire. Il y a également de nombreuses 
formes non-finies et déverbales. Les plus importantes sont les gérondifs qui fonctionnent comme des adverbes et qui prennent leur spécification temporelle en fonction du temps de la proposition principale. Ils expriment la simultanéité ou la postériorité et la subordination.

11 Le système verbal turc peut être caractérisé par deux catégories : une temporelle (passé/ non passé) et une modale (expérience directe/expérience indirecte). La modalité de l'expérience indirecte inclut en même temps déduction, surprise et oui-dire. Le choix d'une des deux formes modales est obligatoire quand on rapporte un événement passé. Le suffixe qui indique l'expérience directe est $\{-\mathrm{DI}\}^{2}$ (parfait testimonial, siglé P.T. par nous). Celui qui exprime l'expérience indirecte ou non-attestée est \{-mIş\} (parfait nontestimonial, siglé P.N.T. par nous) ${ }^{3}$.

Pour illustrer cela, nous pouvons donner les exemples suivants :

\begin{tabular}{|l|l|}
\hline (1) & köpek pencereden düştü \\
\hline & /chien/fenêtre-ABL./tomber-PT.-3sg./ \\
\hline \hline & "le chien est tombé de la fenêtre" \\
\hline
\end{tabular}

\begin{tabular}{|l|l|}
\hline (2) & köpek pencereden düşmüş \\
\hline & /chien/fenêtre-ABL./tomber-PNT.-3sg./ \\
\hline & "le chien est tombé de la fenêtre" \\
\hline
\end{tabular}

12 Si j'ai vu le chien tomber de la fenêtre dans l'histoire de la grenouille, je suis obligé d'employer la forme \{-DI\}; mais si au contraire, je me réfère au fait qu'il est au sol et par inférence il est tombé, ou qu'on m'a raconté qu'il est tombé (ouï-dire), alors je suis obligé de l'exprimer par la forme $\{-\mathrm{mIş}\}$, du fait que l'utilisation référentielle du P.N.T. rapporte un procès passé, terminé, en se fondant sur le résultat. C'est aussi ce dernier qui est le temps des contes et histoires traditionnelles en turc. C'est pourquoi, nous pouvons faire l'hypothèse que nous allons en trouver beaucoup dans les récits des enfants.

Dans le domaine de l'aspect, le morphème \{-Iyor\}, qui est traditionnellement appelé progressif, est plutôt un imperfectif qui sert de présent défini pour les états aussi bien que les événements. Il s'oppose généralement au présent aoristique ou générique $\{-E r\}^{4}$. Les suffixes $\{$-Iyor\} et $\{-\mathrm{Er}\}$ ont été respectivement notés PROG. pour le progressif et AOR. pour l'aoriste. Pour exprimer le temps du futur, le turc a le suffixe (-ECEK). Ce dernier a également une utilisation modale.

Dans les temps passés par contre, le progressif se combine aussi bien avec le parfait testimonial que le parfait non-testimonial pour produire le parfait progressif. Dans le domaine temporel, le suffixe $\{$-DI\} est suffixé à $\{-\mathrm{mI} S\}$, historiquement un participe, pour produire une expérience directe au passé. Nous pouvons également avoir le participe \{-mI ş\} adjoint à sa propre utilisation modale et temporelle. 


\section{Méthodologie}

\subsection{Sujets}

pour la grande majorité nés en France et seulement quelques-uns sont venus en bas âge (avant 2 mois). Leur âge varie de $5 ; 00$ à $10 ; 11$ ans. Les plus petits fréquentent la maternelle et les plus grands sont au CM2. Les enregistrements ont été effectués pendant l'année scolaire 1993-1994, et les sujets ont été choisis à Grenoble et ses environs dans un échantillon représentatif composé de toutes les tendances politiques et religieuses.

Nous présentons dans le tableau ci-dessous le nombre de sujets par tranche d'âge, et la longueur de leur narration mesurée en nombre de propositions :

Tableau 1 : Nombre, âge moyen, nombre total et moyenne de propositions par sujet

\begin{tabular}{|c|c|c|c|c|c|c|}
\hline $\begin{array}{l}\text { Âge } \\
\text { Nombre de sujets }\end{array}$ & $\begin{array}{l}5 \text { ans } \\
\mathrm{N}=14\end{array}$ & $\begin{array}{l}6 \text { ans } \\
\mathrm{N}=14\end{array}$ & $\begin{array}{l}7 \text { ans } \\
\mathrm{N}=16\end{array}$ & $\begin{array}{l}8 \text { ans } \\
\mathrm{N}=17\end{array}$ & $\begin{array}{l}9 \text { ans } \\
\mathrm{N}=17\end{array}$ & $\begin{array}{l}10 \text { ans } \\
\mathrm{N}=15\end{array}$ \\
\hline Âge moyen & 5,4 & 6,4 & 7,6 & 8,4 & 9,6 & 10,6 \\
\hline Amplitude âges & $5 ; 00-5 ; 11$ & $6 ; 00-6 ; 11$ & $7 ; 00-7 ; 11$ & $8 ; 00-8 ; 10$ & $9 ; 00-9 ; 11$ & $10 ; 00-10 ; 11$ \\
\hline Total propositions & 964 & 786 & 825 & 862 & 858 & 602 \\
\hline Moyenne prop./enfant & 69 & 50,5 & 51,5 & 50,5 & 50,5 & 40 \\
\hline $\begin{array}{l}\text { Amplitude } \\
\text { propositions }\end{array}$ & $34-171$ & $31-109$ & $24-80$ & $25-75$ & $34-64$ & $23-64$ \\
\hline
\end{tabular}

Pour permettre de mieux connaître nos sujets, voici quelques indications sur eux et leurs parents.

- L'apprentissage du turc pour les enfants d'immigrés en France se fait jusqu'à l'âge de 7 ans au sein de la famille. A partir de cet âge certains de ces enfants ont la possibilité de fréquenter les cours LCO (Langue et Culture d'Origine) et ce, jusqu'à la fin du collège. A titre indicatif, seulement $38 \%$ de nos sujets fréquentaient ces cours. D'autres enfants ont également la possibilité d'apprendre leur langue d'origine en la pratiquant dans les cours coraniques ( $58 \%$ des sujets) ou des animations organisées en turc par des associations ( $35 \%$ des sujets).

- D'après notre recherche, dans $77 \%$ des familles la langue parlée à la maison est exclusivement le turc, mais $68 \%$ des enfants disent parler le français entre eux. Majoritairement, les pères sont ouvriers et les mères, femmes au foyer. En ce qui concerne la scolarité des parents, $64,5 \%$ des pères ont quitté l'école après le primaire ; $27,5 \%$ ont dépassé le collège, et $8 \%$ ne sont pas allés à l'école. Chez les mères, $61,5 \%$ ont atteint la fin du primaire ; $12,5 \%$ ont dépassé le collège et $26 \%$ ne sont jamais allées à l'école. 


\subsection{Matériel et procédure}

18 Le matériel choisi est un livret d'images sans texte "Frog where are you ?" (M. Mayer, 1969)

5. Ce petit livret comprend 25 images en noir et blanc qui retracent les aventures d'un petit garçon en compagnie de son chien, à la recherche de leur grenouille fugueuse. On peut résumer l'histoire brièvement de la manière suivante : un petit garçon, un chien et une grenouille vivent heureux ensemble. La nuit, pendant que le garçon et le chien dorment, la grenouille s'échappe de son bocal. Le lendemain, le petit garçon et le chien découvrent la disparition de la grenouille et partent à sa recherche. Lors de cette recherche, ils connaissent quelques mésaventures : le petit garçon se fait mordre le nez par une taupe, le chien se fait poursuivre par un essaim d'abeilles ; le petit garçon, effrayé par une chouette, tombe d'un arbre; ils sont projetés par un cerf dans une mare. Ils trouvent finalement des grenouilles dans la mare, et ils rentrent chez eux, en emmenant une (leur?) grenouille avec eux. Il faut noter que l'intrigue est riche en séquences causales et temporelles, en surprises et revirements de situations.

19 Les procédures d'enregistrement ont été les mêmes avec tous les enfants. Nous informons l'enfant en langue turque qu'il va raconter une histoire en turc à une tierce personne d'après un petit livret d'images sans texte. La consigne, donnée en turc, est: "c'est l'histoire d'un petit garçon, d'un petit chien et d'une petite grenouille. Tu vas regarder toutes les images jusqu'au bout puis tu raconteras l'histoire à Y". Ils regardent ensemble les images, le chercheur répond éventuellement à des questions. Une deuxième personne qui est censée ne pas connaître l'histoire entre, et le sujet la lui raconte en regardant les images dans l'ordre chronologique des événements. Et c'est pendant cette phase que la narration du sujet est enregistrée.

20 Après avoir transcrit les enregistrements, nous les avons découpés en propositions (angl. clauses), conformément à la définition de R. A. Berman \& D. I. Slobin (1986) qui considèrent comme clause, "toute unité qui contient un prédicat unifié. Par unifié, nous entendons un prédicat qui exprime une seule situation (activité, événement, état). Dans les prédicats, sont inclus les verbes fléchis et non-fléchis, ainsi que les adjectifs prédicatifs. En général, les propositions comprennent un seul élément verbal. Cependant les infinitifs et les participes qui sont complément d'un verbe modal ou aspectuel sont intégrés à la matrice et considérés comme formant une seule "proposition" ${ }^{\circ}$.

\section{Temps dominants et temps des verbes}

21 Sur le plan développemental, les enfants passent progressivement d'une description image par image à une organisation thématique. Cette dernière peut se réaliser de différentes manières. L'un des moyens est l'établissement et le maintien d'un "temps dominant" ou "temps de base" ou encore "temps d'ancrage". Citons Aksu-Koç (1994) qui dit qu' "un des critères essentiels de la construction d'une narration bien formée est la

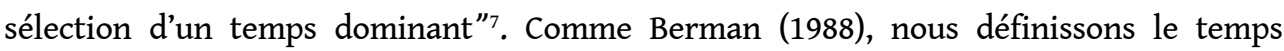
dominant pour chaque narration comme " $75 \%$ ou plus d'occurrences de formes verbales fléchies soit au présent, soit au passé parmi tous les verbes fléchis du texte" ${ }^{\prime}$. Dans le cas contraire, on parle de système mixte. Par ailleurs, pour les histoires en images, plusieurs études portant sur des langues différentes ont montré une préférence généralisée pour le présent, ce qui est certainement lié aux caractéristiques de la tâche et de la procédure, en 
l'occurrence une histoire basée sur des images fixes que le locuteur garde sous les yeux au moment de la production.

De nombreuses études (Bamberg, 1987; Berman, 1988; Berman \& Slobin, 1994, entre autres) ont montré que les plus jeunes enfants ont une tendance à ancrer leurs narrations dans l' "ici et maintenant", signe des problèmes de décontextualisation qu'ils rencontrent. Cette tendance a pour conséquence un emploi massif de formes au présent. Ces mêmes travaux ont mis en évidence une diminution des systèmes mixtes en fonction de l'âge, signe de la maitrise progressive de la constitution d'une narration bien formée.

\subsection{Temps dominants}

Dans les récits en turc, le temps dominant peut aussi bien être le progressif que le parfait testimonial ou le parfait non-testimonial. Et pour Aksu-Koç (1994), "la capacité à suivre la structure de la trame narrative est liée à la tendance à maintenir un temps dominant" 9 . Chez les enfants turcs monolingues, les recherches ont montré qu'entre 2 et 3 ans, ils apprennent très rapidement à utiliser les trois flexions. Autour de 3 ans, ils commencent à opposer les deux formes de parfait en distinguant les modes évidentiels. A 3 ans, les trois formes peuvent être observées dans les narrations, mais elles ne sont pas utilisées suivant une organisation nette des fonctions discursives, et c'est encore plus vrai pour les enfants qui n'adoptent pas un temps d'ancrage. A 5 ans, plus de la moitié d'entre eux ont un temps d'ancrage et produisent un discours cohérent en utilisant les marqueurs aspecto-temporels. A 9 ans et plus, tous les locuteurs manipulent temps et aspect en harmonie avec les règles de l'organisation thématique et cohérente de la narration.

Nous avons voulu savoir ce qu'il en était pour nos sujets. Le tableau 2 présente les temps dominants dans les récits de nos sujets bilingues turc-français et à titre de comparaison, nous donnons dans le tableau 3 ceux des sujets bilingues des Pays-Bas d'après Aarssen (1996). Les chiffres correspondent aux pourcentages, et sont suivis, entre parenthèses, du nombre d'enfants pour chaque catégorie.

Tableau 2 : Pourcentage (et nombre) de sujets en fonction du temps dominant chez les enfants bilingues turc-français

\begin{tabular}{|c|c|c|c|c|c|c|}
\hline $\begin{array}{l}\text { Âge } \\
\text { Nb. de sujets }\end{array}$ & $\begin{array}{l}5 \text { ans } \\
\mathrm{N}=14\end{array}$ & $\begin{array}{l}6 \text { ans } \\
N=14\end{array}$ & $\begin{array}{l}7 \text { ans } \\
\mathrm{N}=16\end{array}$ & $\begin{array}{l}8 \text { ans } \\
N=17\end{array}$ & $\begin{array}{l}9 \text { ans } \\
\mathrm{N}=17\end{array}$ & $\begin{array}{l}10 \text { ans } \\
N=15\end{array}$ \\
\hline Progressif & $7(1)$ & $14(2)$ & $44(7)$ & $41(7)$ & $41(7)$ & $47(7)$ \\
\hline P.T. & & & & & $12(2)$ & $20(3)$ \\
\hline P.N.T. & & & & & $6(1)$ & \\
\hline MIXTE & $93(13)$ & $86(12)$ & $56(9)$ & $59(10)$ & $41(7)$ & $33(5)$ \\
\hline
\end{tabular}

D'après ce tableau, nous pouvons dire que nos sujets bilingues effectuent leurs narrations d'abord avec des temps mixtes. Tous les enfants de 5 ans utilisent de préférence cette catégorie, et le présent progressif n'est choisi comme temps dominant que par un seul sujet. Il en est de même des résultats des 6 ans qui sont sensiblement identiques à ceux 
des 5 ans : nous n'avons que deux sujets qui ont le présent comme temps dominant. Chez les 7 et 8 ans, une moitié des enfants adoptent le présent comme temps dominant. Mais nous n'observons de temps dominants du passé que chez les sujets de 9 et 10 ans. En effet, 3 sujets dans chacune de ces deux tranches d'âge ont fait le choix de raconter le récit au parfait testimonial ou au parfait non-testimonial. Ainsi, la catégorie mixte devient minoritaire pour ne plus représenter que $41 \%$ des sujets âgés de 9 ans et $33 \%$ des 10 ans. Il est donc clair que les temps mixtes dominent largement les récits de nos sujets bilingues jusqu'à 8 ans, et qu'ensuite la tendance s'inverse au profit des temps du passé, même si cette inversion est assez faible par rapport aux bilingues turcs des Pays-Bas, comme le montre le tableau ci-dessous.

Tableau 3 : Pourcentage (et nombre) de sujets en fonction du temps dominant chez les enfants bilingues turcs aux Pays-Bas (Aarssen, 1996) ${ }^{10}$

\begin{tabular}{|l|l|l|l|l|l|l|}
\hline $\begin{array}{l}\text { Âge } \\
\text { Nb. de sujets }\end{array}$ & $\begin{array}{l}5 \text { ans } \\
\mathrm{N}=20\end{array}$ & $\begin{array}{l}6 \text { ans } \\
\mathrm{N}=20\end{array}$ & $\begin{array}{l}7 \text { ans } \\
\mathrm{N}=20\end{array}$ & $\begin{array}{l}8 \text { ans } \\
\mathrm{N}=20\end{array}$ & $\begin{array}{l}9 \text { ans } \\
\mathrm{N}=20\end{array}$ & $\begin{array}{l}10 \text { ans } \\
\mathrm{N}=20\end{array}$ \\
\hline Progressif & $60(12)$ & $30(6)$ & $35(7)$ & $30(6)$ & $50(10)$ & \\
\hline \hline P.T. & & $20(4)$ & $15(3)$ & $10(2)$ & $15(3)$ & $35(7)$ \\
\hline P.N.T. & & $15(3)$ & $10(2)$ & $25(5)$ & $10(2)$ & $30(6)$ \\
\hline \hline MIXTE & $40(8)$ & $35(7)$ & $40(8)$ & $35(7)$ & $25(5)$ & $35(7)$ \\
\hline
\end{tabular}

Chez les enfants bilingues turcs des Pays-Bas, le présent est le temps préféré dès 5 ans. Comme chez les nôtres, les temps du passé sont absents à 5 ans. Par contre, à côté de la domination du progressif jusqu'à l'âge de 9 ans, il y a variation à partir de 6 ans. Le progressif est absent chez les 10 ans. Notons la stabilité de la catégorie mixte, et une utilisation équilibrée des deux temps du passé turc.

Dans l'ensemble la préférence pour les temps mixtes diminue avec l'âge. D'après AksuKoç (1994), la non-utilisation du parfait non-testimonial comme modalité narrative atteste de l'incapacité des enfants à créer une histoire cohérente.

L'exemple (3) montre une utilisation du temps mixte chez un enfant de 9 ans, alors que l'exemple (4) montre celle d'un temps dominant, en l'occurrence le parfait testimonial (l'un des seuls cas de notre corpus) :

\begin{tabular}{|l|l|}
\hline (3) & köpek arı yuvasııı düşürdü, arıların hepsi çıktı, oğlan \\
\hline & ağaca çıktı ve bir deliğe bakıyor \\
\hline & /chien/abeille/foyer-POSS.-ACC./tomber-ACU.-PT.-3sg./ \\
\hline & abeille-Plu.-GEN./tous/sortir-PT.-3sg./garçon/arbre-DIR./ \\
\hline & monter-PT.-3sg./et/un/trou/regarder-PROG.-3sg./ \\
\hline
\end{tabular}


"le chien a fait tomber la ruche, toutes les abeilles sont sorties, le garçon est monté à l'arbre et (il) regarde un trou" $(09 ; 07 i)^{11}$

\begin{tabular}{|l|l|}
\hline (4) & köpek uyurken kurbağa kaçt, çocukla köpek uyandı, \\
\hline & kurbağa yoktu_(...) \\
\hline & $\begin{array}{l}\text { /chien/dormir-AOR.-GER./grenouille/se sauver-PT.-3sg./ enfant-AVEC/chien/lever-REC.- } \\
\text { PT.-3sg./grenouille/ }\end{array}$ \\
\hline & il n'y a pas-PT-3sg./ \\
\hline & "pendant que le chien dormait, la grenouille s'est sauvée, \\
\hline & l'enfant et le chien se sont réveillés, la grenouille, elle n'y était plus" (10;08f) \\
\hline
\end{tabular}

Alors que tous les enfants monolingues turcs ont un temps dominant, plus de la moitié de nos sujets les plus âgés (les 9 et 10 ans) font appel au mélange des temps (catégorie mixte). On peut dire que cela provient de la tâche à réaliser puisqu'il faut raconter l'histoire en même temps que l'on regarde les images, comme l'a montré Erguvanl1Taylan (1987): des sujets adultes devaient raconter une partie d'un film turc immédiatement après l'avoir visionné, et $70 \%$ des narrateurs ont utilisé le progressif comme temps dominant, $10 \%$ l'aoriste et $20 \%$ le parfait testimonial. Notons que nos sujets avaient les images sous les yeux, contrairement à ceux d'Erguvanlı-Taylan.

31 Tous ces résultats suggèrent que le progressif est le temps approprié pour la narration en turc. Aksu-Koç (1994: 335) fait l'hypothèse que le progressif peut être préféré pour raconter des événements vécus de l'intérieur c'est-à-dire vus comme appartenant psychologiquement à soi, alors que le parfait non-testimonial peut être préféré dans les cas où une perspective de distanciation psychologique est choisie.

L'absence d'un temps dominant chez les petits montre qu'ils n'ont pas encore établi une structure narrative unifiée dans laquelle le temps grammatical sert à établir la cohésion et la cohérence du texte. Contrairement aux jeunes, les 7 et 8 ans montrent des signes clairs d'une organisation temporelle puisqu'un peu moins de la majorité (à peu près $40 \%$ ), comme les plus grands, ont un temps dominant, le progressif en l'occurrence. Cela montre qu'ils sont capables de rapporter des événements indépendants des images et qui ne sont pas concomitants avec le temps de la parole. En ce qui concerne le seul sujet de 5 ans chez qui le présent est effectivement le temps d'ancrage, cela provient sans doute de ce que nous avons comptabilisé les existentiels avec le progressif.

Nous venons de voir la différence dans le développement du temps dominant en fonction de l'âge des sujets d'une part, et de leur communauté d'appartenance d'autre part. Ce qui montre que le choix d'un temps dominant a une influence sur le choix des différents temps utilisés dans les productions, comme on va le voir dans les paragraphes qui suivent. 


\subsection{Temps des verbes en turc}

34

Le tableau 5 donne la distribution des différents temps observés dans les narrations de nos sujets. Les temps regroupés sous la catégorie "autres" sont le futur et l'aoriste.

Tableau 5 : Pourcentage des temps des verbes en fonction de l'âge en turc

\begin{tabular}{|l|l|l|l|l|l|l|}
\hline $\begin{array}{l}\text { Âge } \\
\text { Nb. de sujets }\end{array}$ & $\begin{array}{l}5 \text { ans } \\
\mathrm{N}=14\end{array}$ & $\begin{array}{l}6 \text { ans } \\
\mathrm{N}=14\end{array}$ & $\begin{array}{l}7 \text { ans } \\
\mathrm{N}=16\end{array}$ & $\begin{array}{l}8 \text { ans } \\
\mathrm{N}=17\end{array}$ & $\begin{array}{l}9 \text { ans } \\
\mathrm{N}=17\end{array}$ & $\begin{array}{l}10 \text { ans } \\
\mathrm{N}=15\end{array}$ \\
\hline Progressif & 38 & 54 & 50,5 & 60 & 51 & 50,5 \\
\hline Existentiels à l'actuel & 18 & 8 & 11,5 & 11 & 11,5 & 10 \\
\hline Existentiels au passé & 2,5 & 0,5 & 2 & 1 & 13,5 & 2 \\
\hline P.T. & 19,5 & 23,5 & 23,5 & 16,5 & 22 & 29,5 \\
\hline P.N.T. & 16 & 8 & 4,5 & 5,5 & 7,5 & 3 \\
\hline Autres & 4 & 2,5 & 2 & 0,5 & 1,5 & 1 \\
\hline
\end{tabular}

Les résultats de ce tableau semblent au premier abord très surprenants puisqu'il n'apparaît pas clairement de développement dans le domaine du temps en turc. Pour ce qui est du progressif, à part chez les 5 ans, le pourcentage est stagnant à travers les âges, avec un pic d'utilisation pour les 8 ans $(60 \%)$. En ce qui concerne les temps du passé, le parfait non-testimonial est très faiblement employé par tous nos sujets, sauf les 5 ans (16 \%), alors que le parfait testimonial est très employé à tous les âges.

\section{Utilisation des prédicats nominaux et existentiels}

A côté des temps dominants, nous avons également relevé un nombre important d'états, qui sont exprimés par des prédicats nominaux de structure démonstratif + nom ou des prédications avec les formes existentielles var/yok (il y a / il n'y a pas), avec ou sans les suffixes $\{-\mathrm{DI}\}$ ou $\{-\mathrm{mIS}\}$. Voici dans le détail ce que nous obtenons dans nos récits pour ces prédicats nominaux :

Tableau 6 : Pourcentage de prédicats dans les productions chez les bilingues turcs en France

\begin{tabular}{|c|c|c|c|c|c|c|}
\hline $\begin{array}{l}\text { Âge } \\
\text { Nb. de sujets }\end{array}$ & $\begin{array}{l}5 \text { ans } \\
\mathrm{N}=14\end{array}$ & $\begin{array}{l}6 \text { ans } \\
N=14\end{array}$ & $\begin{array}{l}7 \text { ans } \\
N=16\end{array}$ & $\begin{array}{l}8 \text { ans } \\
N=17\end{array}$ & $\begin{array}{l}9 \text { ans } \\
N=17\end{array}$ & $\begin{array}{l}10 \text { ans } \\
N=15\end{array}$ \\
\hline prédicat nom. & 10,5 & 3 & 4 & 5 & 3,5 & 4 \\
\hline var/yok & 7 & 18,5 & 6,5 & 6 & 7 & 6 \\
\hline Total & 17,5 & 21,5 & 10,5 & 11 & 10,5 & 10 \\
\hline
\end{tabular}


Nous constatons que l'utilisation des prédicats nominaux est la plus forte chez les 5 et 6 ans, alors que chez les 7 à 10 ans le pourcentage est faible et stable. Notons que le pourcentage est de $23 \%$ chez les sujets âgés de 3 ans et de $9 \%$ chez les adultes monolingues turcs (Aksu-Koç, 1994). Ce résultat montre à nouveau que le récit des plus petits est davantage influencé par le moment de la parole et de la perception. L'exemple ci-dessous d'un enfant de 5 ans le montre assez bien. Nous avons tout le long du récit de cet enfant la structure : Démonstratif + Nom.

\begin{tabular}{|l|l|}
\hline (5) & bu köpek, bu terlik, bu sandalye \\
\hline & /ceci/chien/ceci/soulier/ceci/chaise/ \\
\hline & "ceci (est) le chien, ceci (est) le soulier, ceci (est) la chaise" (05;06a) \\
\hline
\end{tabular}

Motivées par le contexte, les prédications d'état peuvent être 'juvéniles', comme dans l'exemple (5) : ces prédications simples étiquettent les personnages ou objets présents sur l'image sans véritablement construire une narration, comme le font la majorité des 5 et 6 ans en général. Par contre, de telles prédications peuvent être plus 'matures' si elles sont motivées discursivement et servent à construire un arrière-plan, comme le montre l'exemple suivant d'un enfant de 6 ans :

\begin{tabular}{|l|l|}
\hline (6) & o zaman orda hibou var, o da çocuk da düştü \\
\hline \hline & /cela/temps/cela-LOC./hibou/il y a/lui/aussi/enfant/ \\
\hline & aussi/tomber-PT.-3sg./ \\
\hline \hline & "à ce moment là-bas il y a un hibou, et lui et le garçon est tombé" (06;04f) \\
\hline
\end{tabular}

De telles productions sont plus fréquentes chez les sujets plus âgés. Il s'agit, dans ce cas, de se servir des prédicats nominaux pour exprimer l'arrière-plan des récits, là où, à côté de l'histoire du petit garçon, l'arrière-plan a son importance pour la progression du récit. C'est pourquoi le pourcentage de prédicats nominaux chez les adultes monolingues et chez nos sujets âgés est peu différent. L'exemple (7) extrait du récit d'un enfant de 10 ans le montre également assez clairement. Il utilise la prédication de forme existentielle var (il y a), comme si les mouches n'étaient pas dans le trou, mais en arrière-plan :

\begin{tabular}{|l|l|}
\hline (7) & sonra euh oğlan deliğe bakıyor, bir sürü sinek vardı. \\
\hline & /après/euh/garçon/trou-DIR./regarder-PROG.-3sg./un/ \\
\hline & troupeau/mouche/il y a-PT.-3sg./ \\
\hline & "le garçon regarde dans le trou, il y avait beaucoup de mouches" (10;08k) \\
\hline
\end{tabular}


Ainsi, les prédicats d'état sont provoqués par la perception des images à tous les âges, mais leurs fonctions changent avec l'âge dans la narration.

\section{Les alternances temporelles}

41 Nous reprenons ici la définition et les fonctions des alternances temporelles à Aksu-Koç (1994), qui dit à ce propos que "les alternances temporelles dans la narration ont pour fonctions essentielles d'indiquer les changements dans la perspective temporelle et de parler par 'différentes voix'. L'alternance temporelle permet au locuteur de marquer des distinctions fondamentales entre les événements et de faire un va-et-vient entre le temps de la narration et celui du discours pour des digressions, des apartés et des évaluations" ${ }^{12}$. Ce procédé est rarement présent chez nos sujets, mais très utilisé par des monolingues. Voici l'un des rares exemples d'un enfant de 9 ans : ici, le changement s'effectue du P.T. au P.N.T., et l'évaluation du narrateur provient d'une inférence.

\begin{tabular}{|l|l|}
\hline (8) & sonra köpekle çocuk gördü ki, euh bi kizı sevmiş \\
\hline & /après/chien-AVEC/enfant/voir-PT.-3sg./que/euh/un/ \\
\hline & fille-ACC. /aimer-PNT.-3sg./ \\
\hline & "après le chien et l'enfant ont vu que, euh il a aimé une fille" (09;03m) \\
\hline
\end{tabular}

Comme nous l'avons vu, la majorité des jeunes enfants utilisent un temps mixte et leurs récits présentent de nombreuses alternances temporelles. Ces alternances sont motivées par la nature des activités présentées par l'image et sont donc aspectuelles. Ainsi, la tendance est à un va-et-vient entre la narration et la description. L'exemple (9) a été produit par un enfant de 6 ans chez qui le temps dominant est le progressif, et qui effectue beaucoup d'alternances avec le parfait testimonial. La motivation de ces alternances n'est pas claire, mais cela va conduire l'enfant à associer des activités duratives à un événement concomitant. On peut également parler ici de l'aspect résultatif de l'action constatée par le sujet :

\begin{tabular}{|l|l|}
\hline (9) & ve çocuk deliğe bakıyor. Çocuk düştü. It de koşuyor \\
\hline & /et/enfant/trou-DIR./regarder-PROG.-3sg./enfant/ \\
\hline & tomber-PT.-3sg./chien/aussi/courir-PROG.-3sg./ \\
\hline & "et l'enfant regarde le trou. L'enfant est tombé. Et le chien court" (06;00e) \\
\hline
\end{tabular}

43 Certains enfants utilisent le parfait non-testimonial pour exprimer une information inférée, ou même simplement pour la rétrospection, comme dans l'exemple ci-dessous :

(10) kurbağaya bakıyor, kurbağa yok, gitmiş 


\begin{tabular}{|l|l|}
\hline & /grenouille-DIR./regarder-PROG.-3sg./grenouille/il n'y pas/ \\
\hline \hline & partir-PNT.-3sg./ \\
\hline & "il ( =le garçon) regarde la grenouille, la grenouille n'y est pas, elle est partie" (07 ;11f) \\
\hline
\end{tabular}

L'alternance du P.T. au présent sert dans les évaluations d'information d'arrière-plan en général, alors que l'alternance du progressif au parfait sert d'arrière-plan temporel dans la rétrospection (exemple 11):

\begin{tabular}{|l|l|}
\hline (11) & arılar köpeğin arkasından gidiyor, küçük oğlan ağacın üstüne çıktı \\
\hline & /abeille-Plu./chien-GEN./derrière-POSS.3sg-ABL./ \\
\hline & aller-PROG.-3sg./petit/le garçon/l'arbre-GEN./ \\
\hline & le dessus-POSS.3sg-DIR./monter-PT.-3sg./ \\
\hline & "les abeilles vont derrière le chien, le petit garçon est monté à l'arbre” (07;05k) \\
\hline
\end{tabular}

Chez les sujets monolingues plus âgés, Aksu-Koç relève une autre fonction qui est de marquer la rétrospection d'un événement dominant passé. Il arrive que les narrateurs qui ont choisi un temps dominant et qui le maintiennent aient recours aux propositions adverbiales, complétives et relatives comme modification aspectuelle pour présenter les événements sous différentes perspectives. Nous n'avons pas rencontré de tels emplois chez nos sujets.

\section{Conclusion}

Les résultats de cette étude sur quelques aspects du développement de la temporalité dans les récits turcs des enfants bilingues nous ont révélé que :

- Les 5-6 ans n'ont pas encore la capacité de maintenir un temps dominant d'un bout à l'autre de la narration. Ils présentent les événements les uns après les autres en se fondant sur la succession des images, sans se fonder sur une représentation globale des événements et sans exprimer les motivations des actions. Ils utilisent par ailleurs davantage les existentiels (les verbes être et avoir au présent).

- Les 7-8 ans manifestent un stade intermédiaire entre description et narration. Nous trouvons chez eux des signes d'organisation narrative et temporelle, alors que le progressif reste temps dominant à égalité avec la catégorie mixte, les deux parfaits restant encore absents chez cette tranche d'âge.

- La plupart des récits des 9-10 ans sont de vraies narrations. Les séquences d'images sont transformées en une représentation synthétique d'événements liés temporellement et insérés dans des contextes situationnels particuliers. Nous voyons apparaître les parfaits comme temps dominants, ainsi qu'une nette diminution des temps mixtes.

- Les tableaux 2 et 3 suggèrent que le développement de la narration à travers l'étude des temps dominants en turc se fait plus lentement chez nos sujets bilingues issus de 
l'immigration turque en France par rapport aux enfants autochtones, et aux bilingues des Pays-Bas qui, eux, ont davantage recours à un temps dominant dans leur narration. Plusieurs hypothèses peuvent être évoquées pour expliquer cette tendance :

(i) Le développement "lent" du turc chez nos sujets bilingues comparativement aux bilingues néerlandais turcs est dû à notre avis à la politique d'intégration intensive de la France, avec les moyens consacrés à cet effet (scolarisation des enfants dès le jeune âge, absence de politique de développement des cultures d'origine), alors que les Pays-Bas favorisent l'apprentissage des langues et des cultures d'origine (Aarssen, 1996).

(ii) Le bain linguistique est l'une des conditions favorables à l'acquisition du turc chez les enfants monolingues turcs, par le biais entre autres des récits ou histoires que peuvent leur raconter les parents ou les grand-parents, et celui du milieu scolaire.

(iii) Il faut noter que les productions des sujets monolingues présentent une bonne utilisation des temps du récit dès 3 ans, tandis que nos sujets bilingues, même ceux âgés de 9 ans, ont recours à une utilisation massive des temps mixtes.

(iv) Il faut également tenir compte de la différence de classe sociale entre les monolingues étudiés (de classe sociale élevée) et nos sujets bilingues issus de classe sociale défavorisée : une étude récente (Akinci \& Jisa 1998) a montré que si les différences subsistent par rapport aux monolingues de couche sociale favorisée, les résultats sont très proches et statistiquement non significatifs par rapport à ceux de même origine sociale.

Dans l'acquisition du turc, il semblerait que le paramètre de la classe sociale joue chez nos sujets un rôle important, non seulement dans la production en turc, mais également dans la production de récits en français. En effet, Akinci \& Kern (1997) ont montré dans leur étude comparative d'enfants bilingues turc-français et monolingues français que les habitudes culturelles différentes face à des activités littéraciées (une exposition qualitative et quantitative plus importante chez les monolingues français) font que le retard que connaissent les bilingues persiste jusqu'à dix ans.

\section{BIBLIOGRAPHIE}

AARSSEN, J. (1996) Relating events in two languages: Acquisition of cohesive devices by TurkishDutch bilingual children at scholl age. Studies in Multilingualism 2. Tilbourg : Tilburg University Press.

AKINCI, M.A. \& S. Kern (1997) Développement de la temporalité chez des enfants monolingues et bilingues. Communication présentée au 2ème Colloque Chronos. Bruxelles, Belgique, 9-10-11 janvier 1997 (à paraître dans Chronos n 4).

AKINCI, M.A. \& Jisa, H. (1998) Development of Turkish Clause Linkage in the Narrative texts of Turkish-French bilingual children in France. Paper presented at Ninth International Conference on Turkish Linguistics, Oxford 12-14 August 1998.

AKSU-KOç, A.A. \& Slobin, D.I. (1985) The acquisition of Turkish. In D. I. Slobin (éd.) The crosslinguistic study of language acquisition, vol. 1: The data (pp. 839-878). NJ: Laurence Erlbaum Associates, Hillsdale. 
AKSU-KOÇ, A.A. (1988) The acquisition of aspect and modality: the case of past reference in Turkish. Cambridge University Press, Cambridge.

AKSU-KOç, A.A. (1994) Development of Linguistic Forms: Turkish. In R.A. Berman \& D.I. Slobin (éd.): Relating events in narrative: a crosslinguistic developmental study. $\mathrm{NJ}$ : Laurence Erlbaum Associates, Hillsdale.

BAMBERG, M. (1987) The acquisition of Narratives: Learning to use language. Berlin : Mouton de Gruyter.

BAZIN, L. (1987) Introduction à l'étude pratique de la langue turque. Maisonneuve, Paris.

BERMAN, R.A (1988) On the ability to relate events in narrative. Discourse Process, 11; pp. 469-497.

BERMAN, R.A. \& Slobin, D.I. (1986) Frog story procedures in coding manual: Temporality in discourse. Institute of Human Development, University of California at Berkeley.

BERMAN, R.A. \& Slobin, D.I. (1994) Relating events in narrative: A crosslinguistic developmental study. NJ: Laurence Erlbaum Associates, Hillsdale.

ERGUVANLI, E. (1984) The fonction of word order in Turkish grammer. Presse Universitaire de Californie, Berkeley.

ERGUVANLI-Taylan, E. (1987) Tense variation in Turkish narratives. In H.E. Boeschoten \& L.T. Verhoeven (Éds.), Studies on modern Turkish. Tilbourg: Tilburg University Press, pp. 177-188.

GENCAN, T.N. (1989). Dilbilgisi. Istanbul: Kanaat yayınıarı.

GUENTCHÉVA, Z. (1994) "Interaction entre le médiatif et la personne", in Faits de Langues, 3 : La personne, Paris, PUF. pp. 139-148.

KIBAR, H. (1997) La sémantique des morphèmes verbaux opposés -DI et MIS en turc au passé accompli. Turcica, Revue d'études Turques, Tome n² 29, pp. 245-267.

Koç, N. (1990) Yeni Türk Dilbilgisi. Inkilâp Kitabevi, Istanbul.

MAYER, M. (1969) Frog, where are you? Dial Books for Young Readers. New York.

\section{NOTES}

1. Le turc est essentiellement parlé en Turquie. Mais ce n'est pas le seul endroit où il est pratiqué. Le turc de Turquie fait partie d'un vaste ensemble de "langues turques" (appelées autrefois "turco-tartares"), dont l'expansion géographique est considérable. On compte pas moins de 50 parlers turcs. Ils s'étalent des Balkans à la Sibérie orientale, en passant par l'Asie centrale et le Turkestan chinois. En dépit de leur dispersion géographique, ces parlers forment un ensemble encore très cohérent. Leurs grammaires et le fonds de leurs vocabulaires sont très semblables, les divergences sont surtout d'ordre phonétique.

2. Il est d'usage d'utiliser ce type de symbole $\{-\mathrm{DI}\}$, pour marquer, d'une part, la règle de l'harmonie vocalique, et d'autre part, celle de l'assimilation consonantique. Le symbole I régi par la règle de l'harmonie vocalique des voyelles fermées alterne avec $\mathrm{i}, \mathrm{l}$, $\ddot{\mathrm{u}}$ et $\mathrm{u}$; le $\mathrm{E}$ dans $\{$-EcEK\} peut alterner d'après la règle d'antérieure/postérieure avec e ou a. Le symbole $\mathrm{D}$, alterne lui avec sa correspondante non-voisée $t$ et le $\mathrm{K}$ avec $\breve{g}$.

3. Certains linguistes utilisent d'autres termes pour dénommer ces deux parfaits. Par exemple, $\mathrm{Z}$. Guentchéva (1994) parle de "médiatif" et de "non médiatif" alors que L. Bazin (1987) parle lui de 
"parfait de constatation" et de "parfait de non constatation". Pour une étude détaillée cf. H. Kibar (1997).

4. L'aoriste qui exprime pour L. Bazin (1987: 75) "l'action dans sa généralité, sans actualisation ni limitation de durée" est également appelé "l'atemporel" par le linguiste turc N. Koç (1990) ou "le générique" par T.N. Gencan (1989).

5. ${ }^{1}$ Cette histoire, appelée l'histoire de la grenouille ("Frog story"), est utilisée dans le cadre d'une recherche translinguistique menée sous la responsabilité du Pr. D. I. Slobin de l'Université de Berkeley et du Pr. R. Berman de Tel Aviv et sert de base à la constitution d'un corpus dans une trentaine de langues de par le monde permettant des études comparatives étendues (cf. Berman \& Slobin 1986, 1994).

6. "...any unit that contains a unified predicate. By unified, we mean a predicate that expresses a single situation (activity, event, state). Predicates include finite and nonfinite verbes as well as predicate adjectives. In general clauses will be comprised of a single verbal element; however, infinitives and particles which functions as complements of modal or aspectual verbs are included within the matrix verb as single clause" (Berman \& Slobin, 1986 : 7).

7. "one of the criteria for wellformedness of a narrative is the choice of a consistently favored tense” (Aksu-Koç, 1994 :333).

8. “... as $75 \%$ or more occurrences of either present or past tense verb forms out of the total finite verbs in the text" (Berman, 1988 :484).

9. "The ability to follow the plot structure goes together with the tendency to maintain a dominant tense" (Aksu-Koç, 1994 : 334).

10. L'étiquette 'Progressif' cumule les formes au Présent progressif et les existentiels à l'actuel.

11. Le code attribué à chaque sujet se présente ainsi : 09 indique l'âge en années; 07 l'âge en mois, et $\mathbf{i}$ sert d'identification du sujet dans un groupe d'âge.

12. "The major functions of tense shifting in narrative are to indicate changes in temporal perspective and to speak in "different voices". Tense shifting allows the speaker to make grounding distinctions between events, and to move between narrative and discourse time for digressions, asides, and evaluations" (Aksu-Koç, 1994 :336).

\section{RÉSUMÉS}

L'objet de cette étude est de déterminer quelques aspects du développement de la temporalité en turc (notamment les temps dominants, les temps des verbes, l'utilisation des prédicats nominaux et existentiels et les alternances temporelles) chez les enfants bilingues (turc-français) en France âgés de 5 à 10 ans, dans une tâche de narration à partir du livre d'images Frog, where are you ? de Mayer (1969). Cette étude qui se focalise sur les relations entre formes et fonctions (Berman \& Slobin, 1994) se veut aussi comparative, puisque nous comparons les résultats des bilingues à ceux des monolingues turcs de couche sociale élevée (Aksu-Koç, 1994) et à ceux d'enfants bilingues turc-néerlandais (Aarssen, 1996). Les résultats montrent que les jeunes sujets bilingues (les 5-6 ans) présentent les événements sans lien entre eux et décrivent les contenus des images en utilisant majoritairement un système temporel mixte et des prédicats nominaux. Le progrès réalisé par les 7-8 ans est indéniable : le système mixte est relayé par le présent comme temps de base du récit, mais les temps du passé restent absents. En revanche, chez les 9-10 ans on trouve non seulement le présent comme temps de base du récit, mais aussi les temps du passé. La comparaison des bilingues turc-français avec les groupes témoins montre un certain retard pour 
nos jeunes sujets - retard sans doute lié à des facteurs extra-linguistiques distinguant les deux communautés bilingues (de France et des Pays-Bas), alors que le paramètre de la classe sociale joue un rôle non négligeable pour rendre compte des différences entre les bilingues et les monolingues.

This study aims at characterising some aspects of the development of temporality (especially tenses, narrative tense, use of nominal predicates and tense shifts) in Turkish acquired by Turkish-French bilingual children in France, aged from 5 to 10, in narrative texts elicited from a picture book Frog where are you? (Mayer, 1969). We focus on form and function relations (Berman \& Slobin, 1994) in a comparative perspective : the texts produced by the bilingual children are compared to those produced by monolingual Turkish children from higher socio-economical classes (Aksu-Koç, 1994) and to those of Turkish-Dutch bilingual children (Aarssen, 1996). The results show that the young bilingual children ( 5 and 6 years) do not link the events temporally and rather describe the contents of the pictures, using a mixed narrative tense and above all nominal predicates. The 7-8-years-old show a clear progress : the mixed system is substituted by the present as narrative tense, but past tenses are still lacking. The 9-10-years-old use both present and past as narrative tense. The comparison with the other groups shows some delay in terms of temporality for the younger children (5-6-years-old). This delay is linked to extralinguistic factors which differenciate the two bilingual communities (in France vs. in The Netherlands), while between the bilingual and the monolingual children, differences in socioeconomical status seem to be the main factor.

\section{AUTEUR}

\section{MEHMET-ALI AKINCI}

Laboratoire Dynamique du Langage, (UMR 5596 CNRS) et Université Louis Lumière - Lyon 2 